NOTE

\title{
Radical Reactivities of Vinyl Compounds Bearing Five-Membered Lactone
}

\author{
Takeo SHIMIDZU and Masakazu YoshIKAWA \\ Department of Hydrocarbon Chemistry, Faculty of Engineering, \\ Kyoto University, Kyoto 606, Japan.
}

(Received April 21, 1981)

\begin{abstract}
KEY WORDS Vinyllactone (Five-Membered) / Radical Polymerization / Radical Reactivity Index / Frontier Electron Density / Radical Copolymerization /
\end{abstract}

Five-membered lactone has a moderate chemical reactivity, and is considered to be one of the chemically functional moieties in functional polymers. In the present paper, radical homopolymerizabilities of 2-vinyl-1,4-butyrolactone (1), 3vinyl-1,4-butyrolactone (2), 4-vinyl-1,4-butyrolactone (3), and 3-(4-vinylphenoxy)phthalide (4), and radical copolymerizabilities of these monomers with acrylonitrile (5), a typical radical comonomer, were investigated both experimentally and theoretically. The radical reactivity of 2-vinyl-1,4-butyrolactone (1) was estimated only theoretically, since it has not been synthesized as yet.

\section{CALCULATION}

All calculations were carried out by the INDO MO method. ${ }^{1}$ The radical reactivity index (RRI), the measure of the reactivity of the monomer toward the radical, is defined as,

$$
\mathrm{RRI}=\sum_{i}^{\mathrm{occ}} \frac{\left(C_{r}^{(i)}\right)^{2}}{\lambda-\varepsilon_{i}}+\sum_{j}^{\text {uno }} \frac{\left(C_{r}^{(j)}\right)^{2}}{\varepsilon_{j}-\lambda}
$$

where $C_{r}{ }^{(i)}, C_{r}{ }^{(j)}$ are the coefficients of the $2 p \pi$ atomic orbitals in the $i$ th and $j$ th MOs, respectively, and whose energies are $\varepsilon_{i}$ and $\varepsilon_{j}$, respectively. $\lambda$ denotes the energy of the singly-occupied MO of the attacking radical.

The frontier electron density $\left(f_{r}^{(\mathbf{R})}\right)$ is defined as,

$$
f_{r}^{(\mathrm{R})}=\left(C_{r}^{(\mathrm{HO})}\right)^{2}+\left(C_{r}^{(\mathrm{LU})}\right)^{2}
$$

where $C_{r}{ }^{(\mathrm{HO})}$ and $C_{r}{ }^{(\mathrm{LU})}$ are the coefficients of the $r$ th $2 p \pi$ atomic orbitals in the highest-occupied and lowest-unoccupied MOs, respectively.

\section{RESULTS AND DISCUSSION}

Homopolymerization of both 2 and 3 hardly proceeded. $^{2,3}$ The conversion of 2 was $0.3 \%$ after 7 days and that of $\mathbf{3}$ was even less than that of $\mathbf{2}$. These results lead to the conclusion that the homopolymerization of $\mathbf{2}$ and $\mathbf{3}$ are difficult. On the other hand, the radical homopolymerization of 4 proceeded in a manner similar to ordinary vinyl monomers. ${ }^{4}$ This means that 4 has a reactivity higher than either $\mathbf{2}$ or $\mathbf{3}$ and that $\mathbf{2}$ is more reactive than $\mathbf{3}$. The monomer reactivity ratios (MRR) of the radical copolymerization of $\mathbf{2}$ and $\mathbf{5}$, and that of $\mathbf{3}$ and $\mathbf{5}$ are shown in Table I. Of these two copolymerizations, the propagations were influenced only by the terminal monomer unit and are interpreted as a terminal model. Of the radical copolymerization of 4 and 5 , the propagation was influenced not only by the terminal monomer unit but also by the penultimate unit of the growing chain since 4 is very bulky. This propagation is interpreted on the basis of the penultimate model. The monomer reactivity ratios $r_{4}=0.23, r_{4}{ }^{\prime}=0.41, r_{5}=0.08$, and $r_{5}{ }^{\prime}=0.11$

Table I. Monomer reactivity ratios of the radical copolymerization of $\mathbf{2}$ and $\mathbf{3}$ with $\mathbf{5}$

\begin{tabular}{ccc}
\hline Monomer & \multicolumn{2}{c}{ MRR } \\
\hline 2 & $r_{2}=0.058$ & $r_{5}=4.6$ \\
3 & $r_{3}=0.004$ & $r_{5}=4.4$ \\
\hline
\end{tabular}


Table II. Radical reactivity indices (RRI) and frontier electron densities $\left(f_{r}^{(\mathbf{R})}\right)$ for $1,2,3,4$, and 5

\begin{tabular}{ccc}
\hline Position & RRI $^{*}$ & $f_{r}^{(\mathrm{R})}$ \\
\hline $\mathrm{a}$ & 2.90 & - \\
$\mathrm{b}$ & 2.98 & - \\
$\mathrm{c}$ & 2.59 & 0.360 \\
$\mathrm{~d}$ & 2.78 & 0.458 \\
$\mathrm{e}$ & 2.51 & 0.442 \\
$\mathrm{f}$ & 2.77 & 0.597 \\
$\mathrm{~g}$ & 2.51 & 0.154 \\
$\mathrm{~h}$ & 2.91 & 0.356 \\
$\mathrm{i}$ & 2.50 & 0.525 \\
$\mathrm{j}$ & 2.82 & 0.786 \\
\hline
\end{tabular}

* RRI was calculated, taking the value $\lambda=-0.15$ a.u.<smiles>O=CC1CCOC1=O</smiles><smiles>[Z]</smiles><smiles>C=CC1COC(=O)C1</smiles><smiles>C=CC1CCC(=O)O1</smiles><smiles></smiles>

were obtained, ${ }^{4}$ on the basis of the chemical composition and triad fraction analysis of the copolymer. Using these results and the published $Q$ and $e$ values of $5,{ }^{5} Q_{2}, e_{2}, Q_{3}$, and $e_{3}$ were calculated to be $Q_{2}=0.033, e_{2}=0.053, Q_{3}=0.012$, and $e_{3}=-0.81$. These values imply that the reasonance stabilization of $\mathbf{2}$ and $\mathbf{3}$ are low, but the $\pi$-electron density of $\mathbf{2}$ is relatively low and that of 3 is high. The high $\pi$ electron density of $\mathbf{3}$ is considered to be attributable to the ether oxygen atom adjacent to the vinyl group.

In Table II, RRI and $f_{r}^{(\mathbf{R})}$ are shown. As for $f_{r}^{(\mathbf{R})}$ of 2, 3, and 4, the coefficients of the vinyl carbon $2 p \pi$ atomic orbital in the highest-occupied and lowestunoccupied MOs are small, but the largest coefficient of the vinyl carbon $2 p \pi$ atomic orbital in the occupied MO exists below the highest-occupied $\mathrm{MO}$, and the largest one in the unoccupied MO exists above the lowest-unoccupied MO, respectively. Using the coefficients of these vinyl carbon $2 p \pi$ atomic orbitals, $f_{r}^{(\mathbf{R})}$ was calculated. But, in the case of 1 , the coefficients of the vinyl carbon $2 p \pi$ atomic orbital are dispersed, and the inability to identify orbitals made evaluation of $f_{r}^{(\mathbf{R})}$ impossible.
As for RRI, the orbital energy of the radical, $\lambda$, which attacks the monomer, is assumed to be -0.15 a.u. Both the RRI and $f_{r}^{(\mathrm{R})}$ values indicate that the reacting position of these five monomers toward the growing chain radical is situated mostly at the $\beta$ carbon of the vinyl group. Accordingly, the polymerization proceeds via a head-to-tail mechanism and the growing chain terminals are,<smiles>CCC1CCOC1=O</smiles>

6<smiles>CCC1COC(=O)C1</smiles>

7<smiles>CCC1CCC(=O)O1</smiles>

8<smiles>CCc1ccc(OC2OC(=O)c3ccccc32)cc1</smiles>

The growing chain terminal structures, 6,7 , and $\mathbf{8}$, are readily converted into allylic radicals by losing 2-hydrogen of 6, 3-hydrogen of 7, and 4-hydrogen of $\mathbf{8}$, respectively. In addition, there is the possibility of intermolecular hydrogen migration, meaning that these growing chain terminals may also subtract another 2-hydrogen of 1, 3-hydrogen of 2, and 4-hydrogen of 3. Accordingly, in the radical copolymerization of $\mathbf{2}$ and $\mathbf{5}$, and $\mathbf{3}$ and $\mathbf{5}$, the degrees of polymerization decreased with the content of 2 in the poly $(2-c o-5)$ and that of 3 in the poly(3-co-5), respectively.

From RRI, the radical reactivities of these three 1,4-butyrolactone derivatives decreased in the following order; $1>\mathbf{2}>\mathbf{3}$. This order coincided with the experimental data, but not the order in $f_{r}^{(\mathrm{R})}$. RRI predicts that $\mathbf{1}$ is a highly reactive monomer compared with the other 1,4-butyrolactone derivatives, though 1 has not been synthesized. These trends are not altered by the assumption of $\lambda$ 's being -0.05 , $-0.10,-0.20$, or -0.25 a.u.

Since RRI is calculated by considering not only the coefficient of the vinyl carbon $2 p \pi$ atomic orbital but also its orbital energy, it is possible to assume both the reactivity and reacting position in vinyl compounds, even though coefficients of vinyl car- 
bon $2 p \pi$ atomic orbitals are dispersed, as in the case of 1 reported in the present paper.

Acknowledgement. The authors are grateful to the Data Processing Center of Kyoto University for kindly allowing us to use FACOM M190 and M200 Computer.

The authors are also indebted to Dr. H.Fujimoto and Mr. N. Koga for their stimulating discussion on the theoretical calculation. This work was supported by a Grant-in-Aid from the Ministry of Education, Science and Culture of Japan.

\section{REFERENCES}

1. J. A. Pople and D. L. Beveridge, "Approximate Molecular Orbital Theory," McGraw-Hill, New York, N.Y., 1970.

2. T. Shimidzu, M. Yoshikawa, H. Chiba, and A. Murakami, Makromol. Chem., 178, 1923 (1977).

3. T. Shimidzu, M. Yoshikawa, M. Hasegawa, and H. Fujimoto, Polym. J., 11, 607 (1979).

4. T. Shimidzu, M. Yoshikawa, and B. Ohtani, Macromolecules, 14, 506 (1981).

5. L. J. Young, J. Polym. Sci., 54, 411 (1961). 